\title{
Conservative removal of small pituitary tumours: is it justified by the pathological findings?
}

\author{
P H I L I P W R I G H T S O N \\ From the Department of Neurology and Neurosurgery, Auckland Hospital and The Department of \\ Surgery, University of Auckland, Auckland, New Zealand
}

SU M M AR Y Operation by the trans-sphenoidal route allows removal of small pituitary adenomata with conservation of normal gland. Histological examination of tissue obtained at operation and necropsy in 73 cases showed that surgical methods at present in use are likely to leave tumour behind in the pituitary gland and in the dura mater of the pituitary fossa. The clinical significance of these findings will only become evident after following patients for an extended period, but there appears to be a strong indication for routine postoperative radiotherapy.

The development of the trans-nasal operation for pituitary adenomas has made it possible in suitable cases to remove the tumour and preserve normal gland. The ability to diagnose small secreting tumours has provided such cases, and the early results have been good, with remission of symptoms of oversecretion and retention of pituitary function in many cases (Williams et al., 1975, Hardy et al., 1976). However, close examination of what is done at operation and of the criteria of endocrine assessment postoperatively raises doubt whether the long-term results will be as satisfactory. The growth of pituitary tumours is slow and the situation will not be clear for some years; in the meantime management policy needs constant review and it is important to look for ways of anticipating the final judgement.

The critical issues are whether conservative removal of adenomas is complete enough to abolish abnormal secretion and to prevent recurrence; and whether radiotherapy should be given postoperatively as a routine, or only when there are specific signs that operation has been incomplete. The question whether the remaining normal gland, subject to the continuing influence of the hypothalamus, is liable to produce further tumours will not be considered here.

Complete removal of the tumour has been claimed if there is clinical remission and if hor-

Address for reprint requests: Auckland Hospital, Private Bag, Auckland 1, New Zealand.

Accepted 14 October 1977 mone levels return to normal. However, though the reduction of clinical signs after operation is often dramatic, it may be difficult to be sure that they have disappeared completely, especially if there are residual tissue changes such as occur in acromegaly. Postoperative hormone levels are subject to a similar uncertainty. In judging their significance it is necessary to allow for the contribution from the remaining normal gland, which may entirely mask the continuing autonomous secretion from a small amount of residual tumour.

Faced with these doubts, a histological assessment of the adequacy of conservative removal would be useful. The opportunity for a complete assessment postoperatively is, fortunately, rare, but useful conclusions can be drawn from material removed at both conservative and radical operations, and from necropsy of patients with pituitary tumours. From the examination of this material, some answers may be given to the questions the surgeon asks when he attempts the conservative removal of an adenoma. Firstly, which is tumour and which is gland? Tumour is usually paler than gland, but the important difference is that of consistency, tumour being soft and gland firm. The histologist will see this as a difference of cellularity and the amount of a connective tissue stroma.

The second question is whether there is a plane of cleavage between tumour and gland, and whether dissection along this plane is likely to result in complete removal of the tumour. To the histologist, a definite plane of cleavage will show as a sharp transition of cellularity and stroma 
content on passing from tumour to gland. The change of consistency which guides the surgeon may be exaggerated by increase of connective tissue in the gland at the junction, partly due to compression of its stroma and partly to the formation of new connective tissue. If removal is to be complete, there must be no tumour on the gland side of this plane of dissection.

Lastly, having removed the adenoma, does the bed in which it lay contain tumour cells? The remaining normal gland will be examined for tumour cells, but dura mater also is often in contact with tumour, and it too must be searched for invasion (Shaffi and Wrightson, 1975).

If these three questions can be answered, judgement of the effectiveness of conservative removal of a pituitary adenoma may be possible.

\section{Materials and methods}

Histological material was examined from 73 patients presenting from 1967 onwards with clinical features of pituitary adenoma. Forty-four patients had acromegaly, four had Cushing's disease (three with Nelson's syndrome), two presented with amenorrhoea and galactorrhoea, and 23 had no clinical evidence of increased hormone secretion. In 64 cases the specimens were obtained at operation and in nine at necropsy.

Biopsy material consisted of tumour, fragments of normal gland, and, where possible, pieces of the diaphragma sellae or dura mater from the anterior wall of the fossa. It was usually fixed in formalin, embedded in paraffin, and cut at several levels.

Necropsy material was fixed in formalin. In eight cases the sphenoid and sella turcica were removed entirely, decalcified, and serial or stepserial sections cut in the coronal plane.

All sections were stained with haematoxylin and eosin (H \& E) and with periodic acid-Schiffhaematoxylin-Orange G (PAS/Hx/OG). Masson trichrome, Heidenhain-Azan, and PAS/Hx/OG/ Light green stains were used on some sections.

Black and white prints were made from photographs on Ilford Pan F film: with $\mathrm{H} \& \mathrm{E}$ staining a light green filter was used, and with other stains usually no filter.

\section{Results}

In 24 of the 73 cases enough normal gland was present to allow comparison of the amount of stroma in the normal gland with that in the tumour. In all cases most of the tumour was highly cellular and contained very little stroma.
In four cases there were areas of tumour, measuring $0.5 \mathrm{~mm}$ to $1 \mathrm{~mm}$ across on the slide, with about the same amount of stroma as the gland, and in four further cases some areas showed a good deal more stroma than normal gland (Figs. 1 and 2). It is reasonable to conclude, therefore, that soft areas encountered at operation are tumour, but that tumour may be present in areas which are indistinguishable by their consistency from normal gland.

In 13 of the 24 cases in which normal gland was present, there were areas in which the boundary between tumour and gland could be seen without artefact for a reasonable distance, usually between $0.5 \mathrm{~mm}$ and $1 \mathrm{~mm}$ on the slide. Passing from tumour to normal gland, the boundary was usually marked by a diminution of cellularity and an increase in stroma (Fig. 1). The tissue of the gland was often somewhat compressed at the junction, with the cells ranged in rows parallel to it. In two of the 13 cases there was a considerable accumulation of connective tissue in the gland at the boundary. In one of these there was further tumour on the far, or gland, side of the connective tissue (Figs. 3 and 4 ). The change in cell morphology at the apparent boundary was examined. In two of the 13 cases the change was sharp throughout and coincided with the boundary (Fig. 1). In eight cases the general line of the boundary was apparent, but there was considerable cellular interchange across it (Fig. 5). In three cases there was no real boundary to be seen, cells of tumour and gland mingling over a wide area (Fig. 6). It is again reasonable to say that, though in most cases the plane of surgical dissection will coincide with the boundary of the tumour, in some cases tumour cells will be present in the normal gland beyond this plane, and that in others the distinction between tumour and gland will be lost and no plane will be detectable.

In 41 cases parts of the dura mater from the tumour bed were examined. In 23 cases there was definite invasion by tumour, six of these being classed as gross (Fig. 7).

Four of the nine necropsies were upon patients who had been diagnosed in life as having small secreting tumours and who had died from causes other than their pituitary tumour. In one case, the adenoma was uniform in structure, sharply circumscribed, and without invasion of normal gland or dura mater, as in Fig. 1. In one, the boundary between tumour and gland was diffuse (Fig. 6). In two cases there was tumour tissue outside the sellar dura mater, in one in the cavernous sinus and in the other in the bone of the posterior clinoid process. 


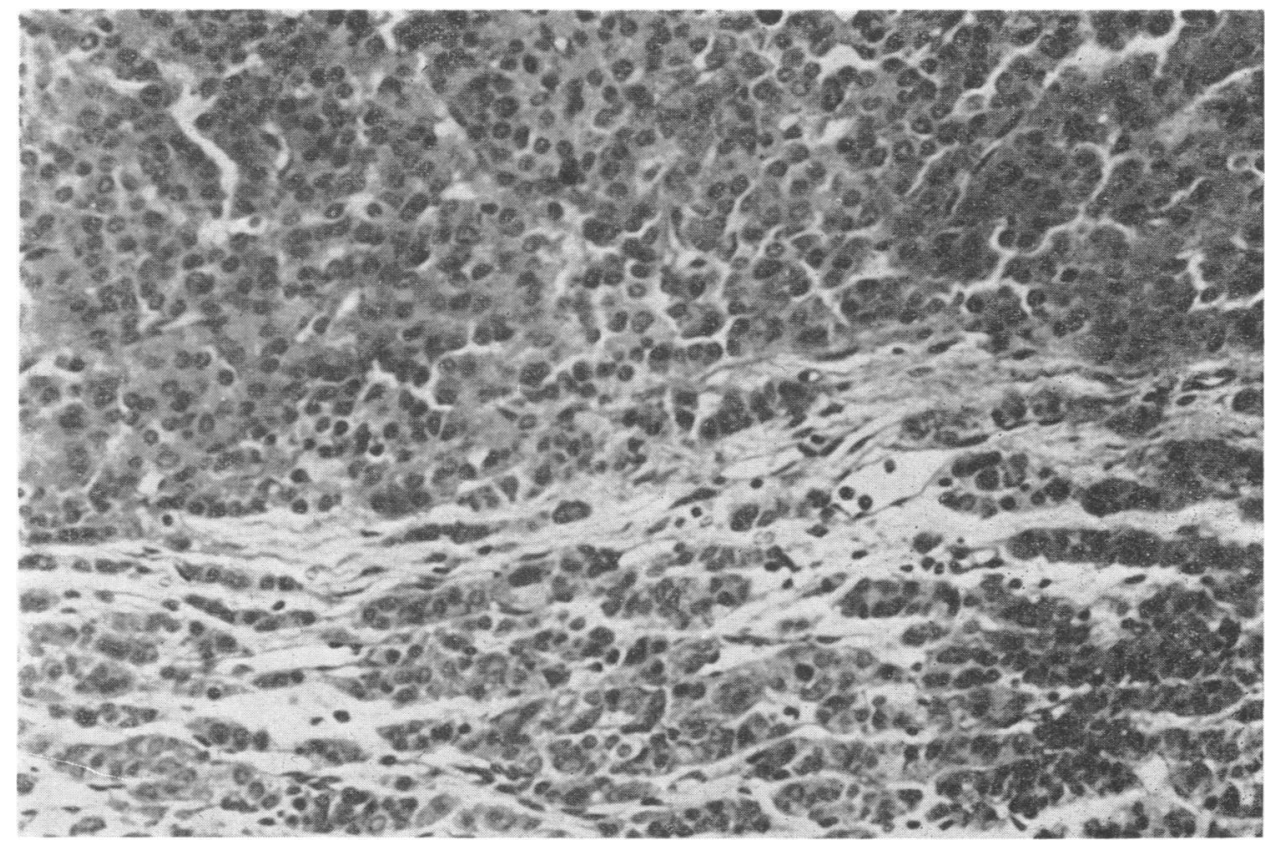

Fig. 1 Acromegaly PH. Operative specimen-adenoma above, normal gland below.

$P A S / H x / O G \times 250$.

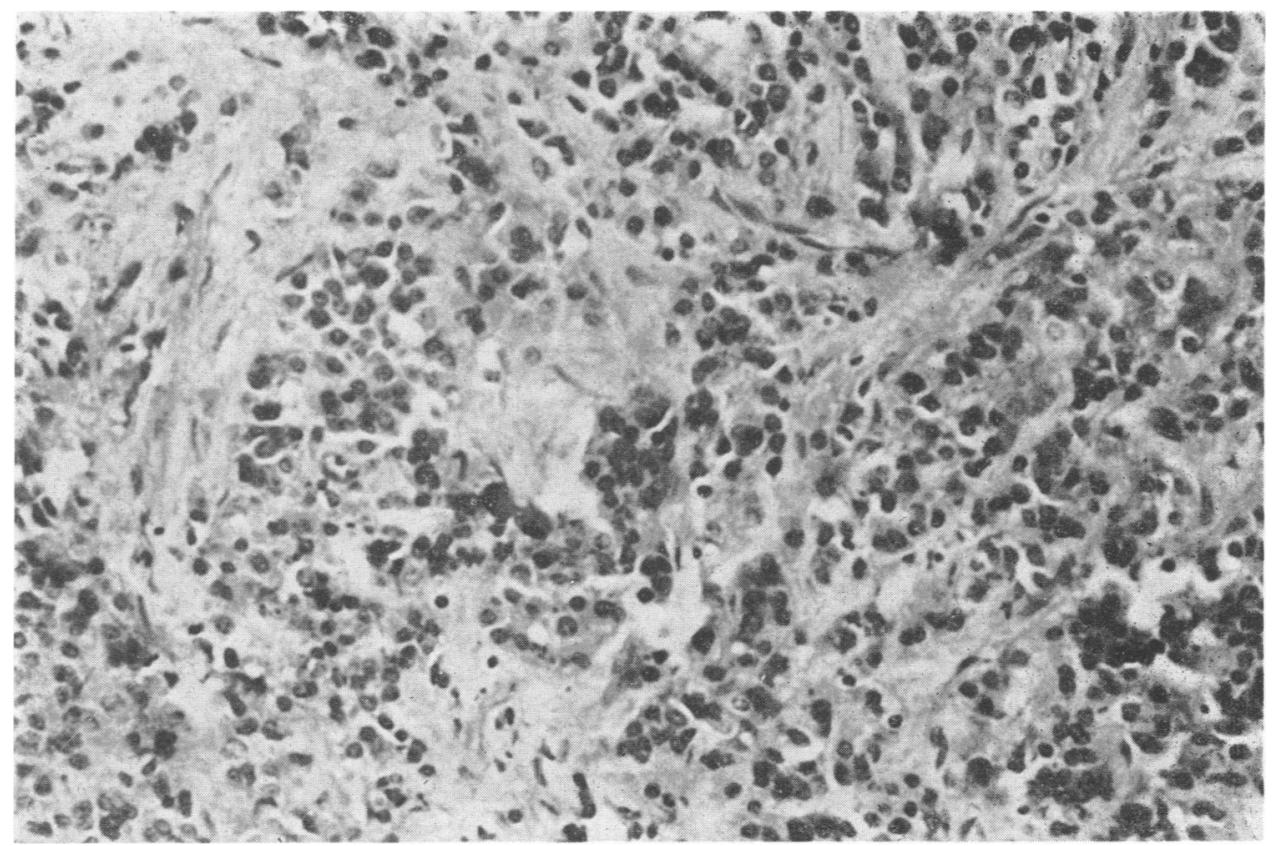

Fig. 2 Acromegaly PH. Same slide as Fig. 1. Area of adenoma showing marked increase of stroma. PAS/Hx/OG $\times 250$. 


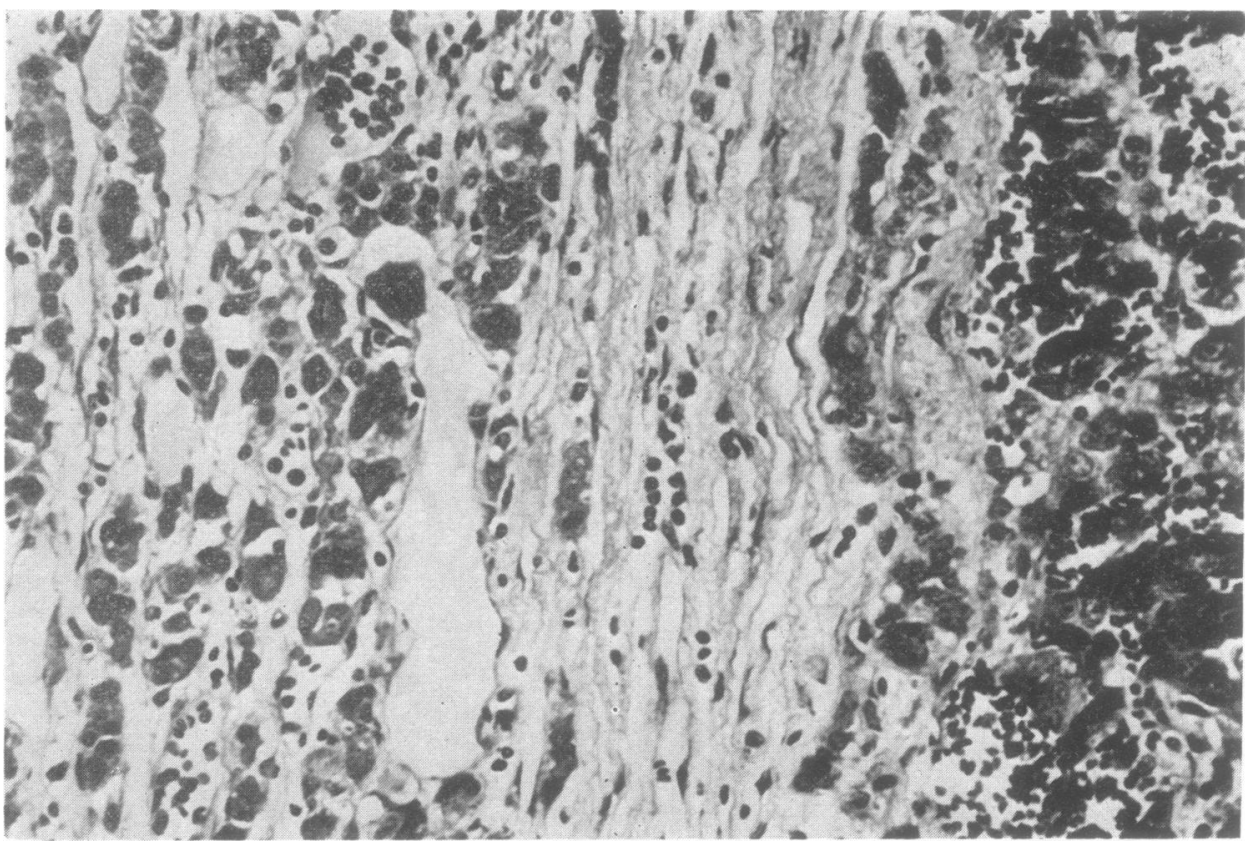

Fig. 3 Acromegaly AK. Operative specimen-adenoma on right, normal gland on left, separated by zone of collagenous tissue. No tumour cells seen in gland tissue. Masson trichrome $\times 250$.

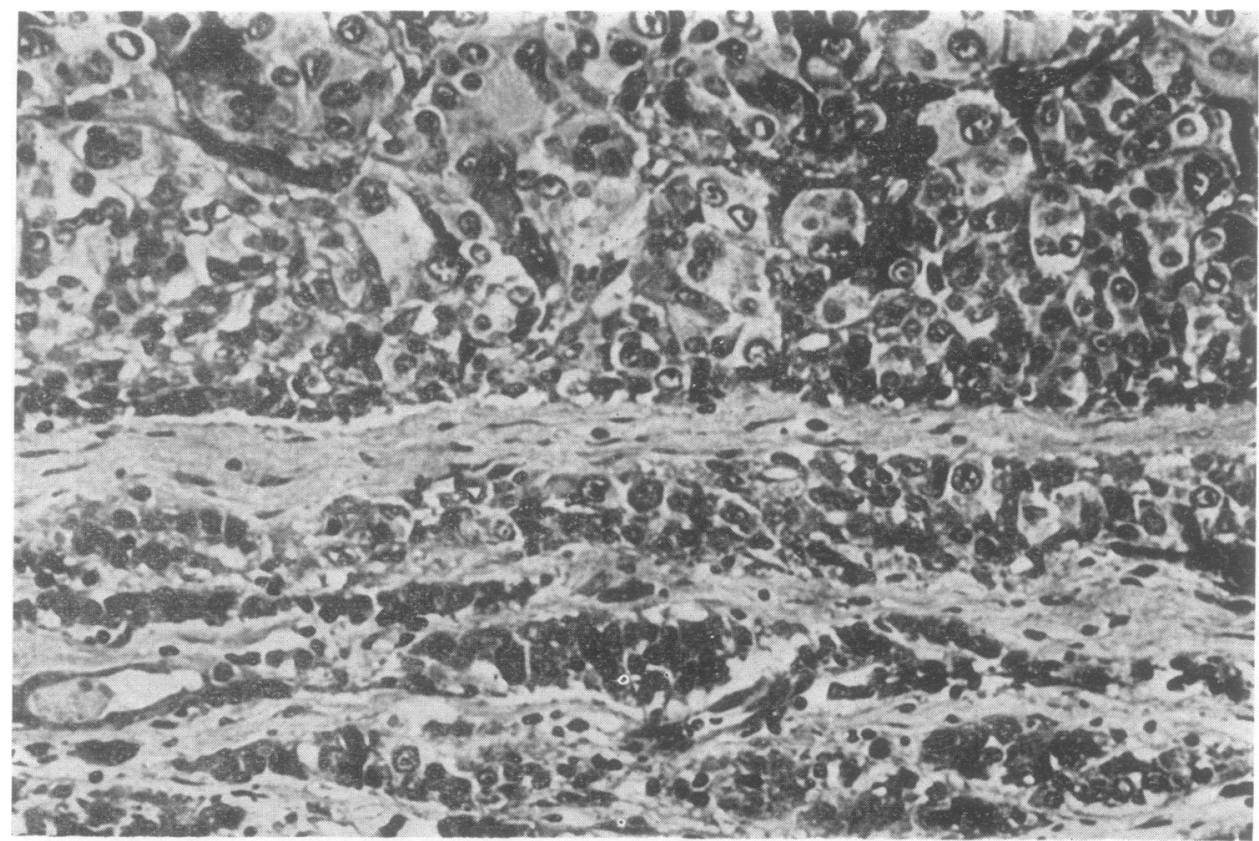

Fig. 4 Acromegaly AK. Same patient as Fig. 3, different level of section. Adenoma above, normal gland below, separated by collagenous tissue. Tumour present on the gland side of collagenous tissue. Masson trichrome $\times 250$ 


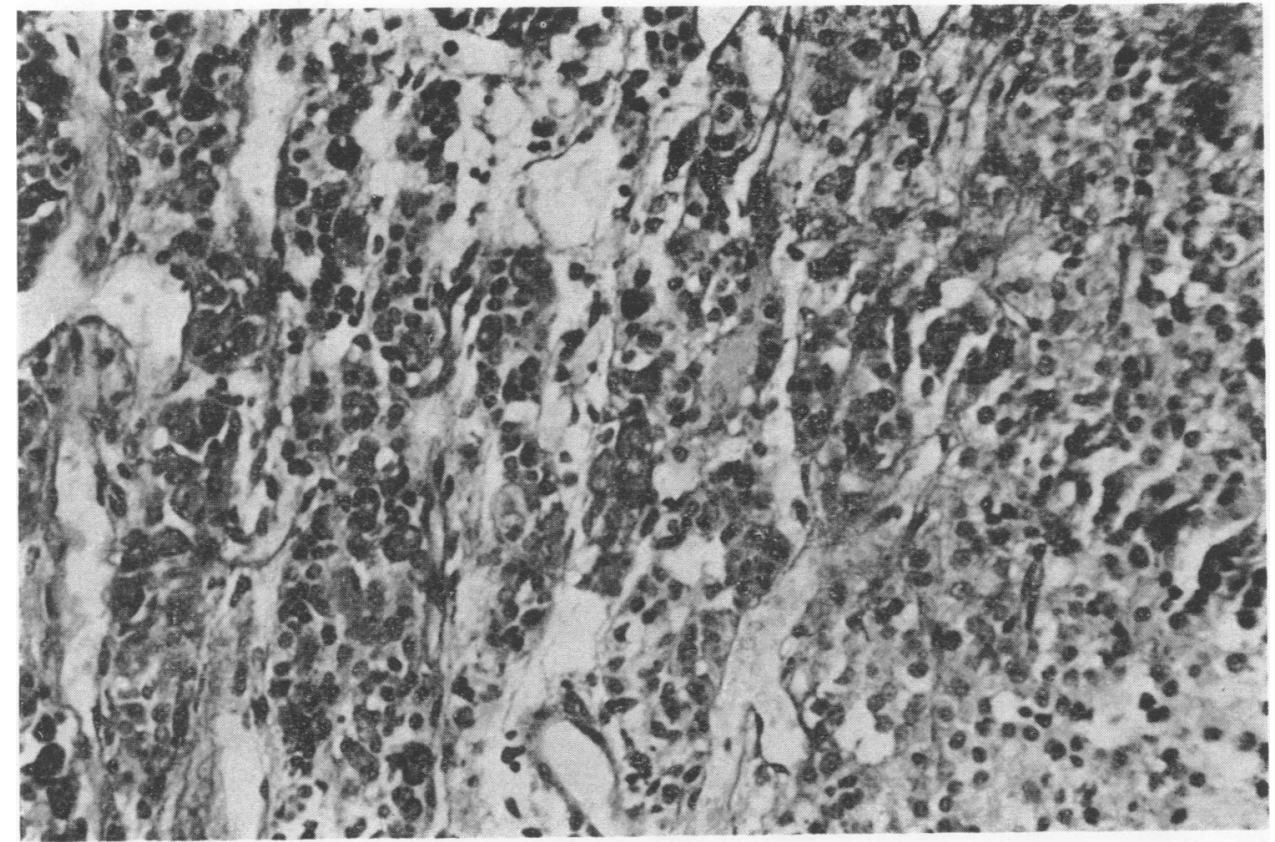

Fig. 5 Acromegaly AP. Operative specimen-adenoma on right occupying one-third of figure, normal gland on left. The position of boundary can be estimated, but there is no definite line. $P A S / H x / O G \times 250$.

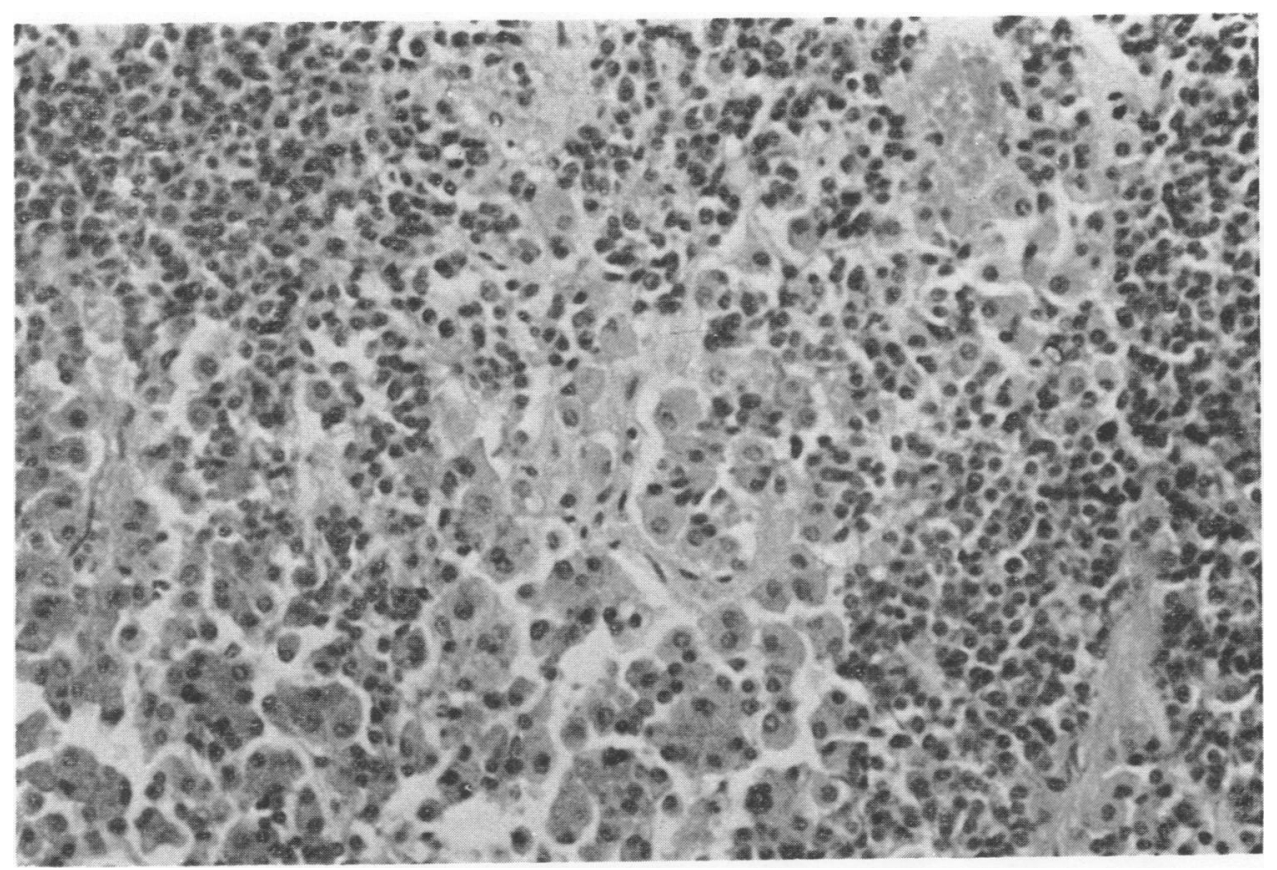

Fig. 6 Cushing's disease CT. Necropsy specimen, death five days after adrenalectomy. Tumour cells have small darkly staining nuclei. Main mass of tumour to the right of the area photographed. Note absence of boundary with normal gland and free infiltration. $H$ and $E \times 250$. 


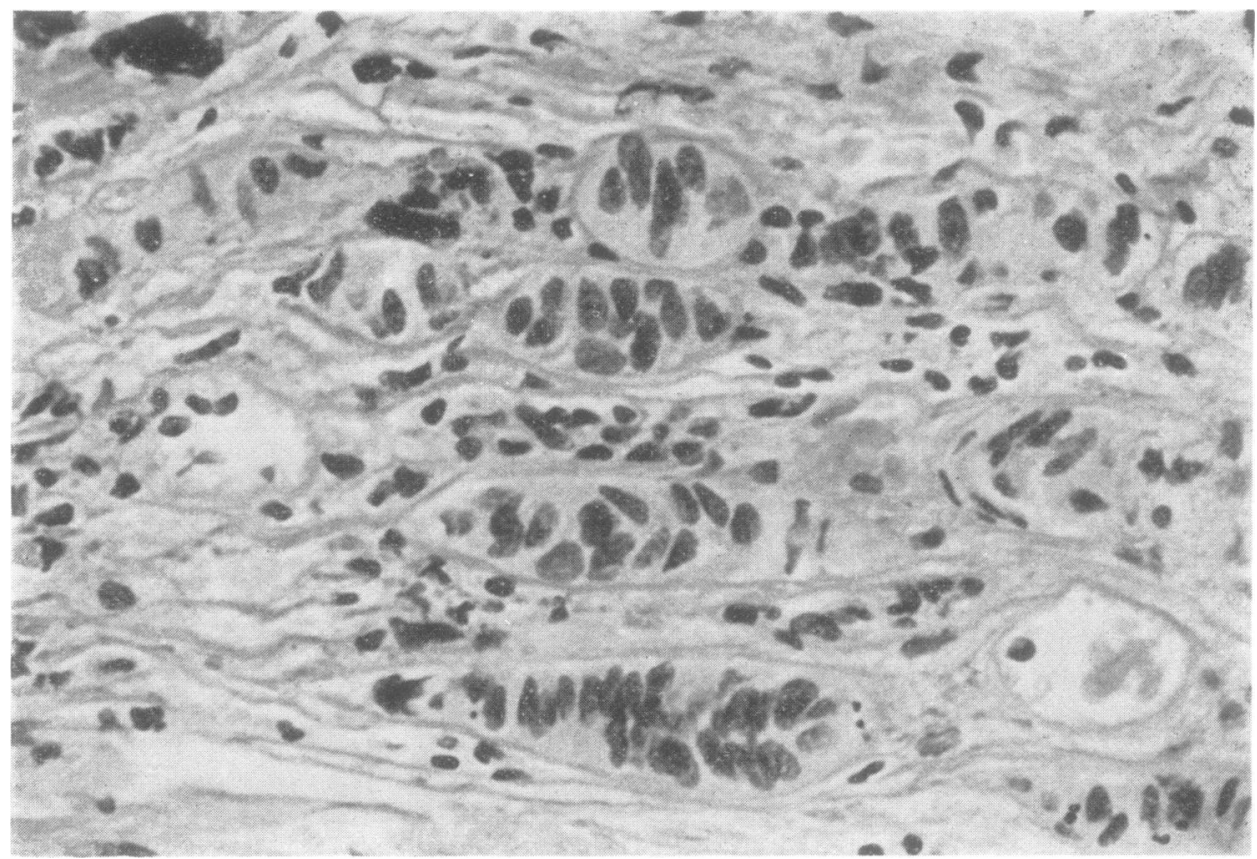

Fig. 7 Non-secreting adenoma BH. Operative specimen of diaphragma sellae. The connective tissue has been infiltrated by spindles of tumour cells. $H$ and $E \times 500$.

It is reasonable to conclude, therefore, that in over $50 \%$ of cases tumour will have spread from its bed into the dura mater of the pituitary fossa and that, even in the case of small and slowly growing tumours, the bone of the sella turcica or the cavernous sinus may be involved.

\section{Discussion}

These findings have given definite answers to the three questions posed in the introduction. They show that to make sure of removing the source of abnormal secretion and to prevent regrowth, the surgeon must excise as widely as he can the bed in which the tumour lay, as well as the tumour itself. In doing this he will be constrained by two factors, the need to preserve normal gland, and the difficulty of removing the dura mater which has been in contact with the tumour.

The need to preserve normal gland will vary from patient to patient. In a middle-aged man with acromegaly who already shows some pituitary deficiency, the advantage of retaining normal gland tissue will be outweighed by that of complete clearance of the sellar contents. Replacement therapy for the postoperative hypopituitarism should present no problems. On the other hand, in the case of a young woman who complains of infertility, with amenorrhoea and galactorrhoea, it is obligatory in removing her prolactin secreting adenoma to preserve pituitary function, even at the expense of leaving some tumour.

The ability to preserve the gland depends on making the distinction between it and tumour. At operation, the first step is to remove the soft part of the tumour. This will expose some areas of apparently clean separation from normal gland. There will be others where there is tissue which appears abnormal, and this must be removed piecemeal, remembering that parts of the tumour may have the same consistency as gland. We have seen that the resulting surface, even in the areas where the separation appeared clean, cannot be taken to be free of tumour, and there is reason to use an agent such as Zenker's fluid in the hope of destroying the superficial half millimetre of tissue. If there is condensation of connective tissue at the boundary, it will be easier to separate tumour tissue from within it. Bergland (1975) has illustrated from Hardy's necropsy material a microadenoma with a "capsule" presumably of this sort. However, tumour may have penetrated this capsule ( $c f$ Fig. 4) and the plane of excision should be outside it and not within, as Bergland 
suggests. Where wide excision seems likely to compromise normal gland, biopsy and frozen section may help to distinguish normal from abnormal tissue (Hardy, 1973). However, with a small fragment of tissue whose structure is distorted by removal it is difficult for the pathologist to be sure that tumour is not present, and the number of areas which can be sampled is limited, so that the manoeuvre may not be of great benefit.

The other element of the tumour bed, the dura mater, lines the bone of the sella turcica and is folded to form the diaphragma sellae and the walls of the cavernous sinus. Any adenoma which grows to involve the surface of the gland will come into contact with dura mater, and large tumours will remain invested by it as they arise out of the fossa or expand into the sphenoidal sinus. As Hardy (1975) points out, this was referred to in older literature as the "capsule" of the tumour, and it was sometimes recommended that it should be removed. As it forms the wall of the cavernous sinus and merges with the adventitia of the internal carotid arteries, this is plainly impracticable. Thus, though it has been shown that the dura mater is invaded by tumour in at least $50 \%$ of cases, no practical surgical measure is available for removing it.

It is necessary then for the surgeon to accept the anatomical limits to the tissue that he may remove, and the endocrine dictates of what he must leave, and that some tumour is likely to remain after the widest excision that these limits allow. The effects of such persistent tumour will depend on its mass, its biological behaviour, and its sensitivity to radiotherapy. Decisions on further treatment are relatively straightforward if the mass of the tumour is sufficient for it to be detected by persistence of abnormal hormone secretion. There appears to be a consensus that unless there should be a special reason for reoperation, radiotherapy should be given. This is likely to prevent regrowth of tumour, and there is a reasonable chance that, with time, abnormal hormone secretion will decrease. If blood hormone levels are normal, however, there is no proof available of persisting tumour; the consensus appears to be to withhold radiation until such proof appears (Hardy, 1975; Williams et al., 1975). The present findings suggest, however, that though the surgical clearance may appear to have been good, some tumour is likely to remain, though there may not be sufficient for its hormone output to be readily detected. Since the results of radiotherapy should be best when the tumour mass is smallest, routine postoperative radiation seems indicated. If it is withheld until abnormal hormone levels provide evidence of regrowth, the wheel has turned full circle and the patient is in the same situation as when he first presented with his illness; radiotherapy would not have been expected to cure him then.

Though the histological observations here are definite, conclusions about their clinical relevance can only be tentative. Only careful observation of several series of patients over a number of years will show whether conservative removal of small pituitary tumours is a safe and adequate procedure. Until this time, the pathological limitations must be kept in mind and precautions, such as careful attention to surgical detail and the use of adjuvant radiotherapy, should be taken to circumvent them.

I am grateful to Associate Professor J. F. Arthur for allowing me access to the material of the Department of Pathology, Auckland Hospital; to Professor E. M. Nanson for laboratory facilities; and to Mr R. Paterson, Mrs K. Speak, and Miss A. Richards for their histological preparations. The work has been done in collaboration with the Department of Endocrinology, Auckland Hospital, and was supported by a grant from the Hackett Memorial Trust.

\section{References}

Bergland, R. M. (1975). Pathological considerations in pituitary tumours. Progress in Neurological Surgery, 6, 62-94.

Hardy, J. (1973). Transsphenoidal surgery of hypersecreting pituitary tumours. In Diagnosis and Treatment of Pituitary Tumours. Edited by P. O. Kohler and G. T. Ross. Excerpta Medica: Amsterdam.

Hardy, J. (1975). Trans-sphenoidal microsurgical removal of pituitary micro-adenoma. Progress in Neurological Surgery. 6, 200-216.

Hardy, J., Somma, M., and Vezina. J. L. (1976). Treatment of acromegaly: radiation or surgery? In Current Controversies in Neurosurgery. Edited by T. P. Morley. W. B. Saunders: Philadelphia.

Shaffi, O. M., and Wrightson, P. (1975). Dural invasion by pituitary tumours. New Zealand Medical Journal, 81, 386-390.

Williams, R. A., Jacobs, H. S., Kurtz, A. B., Millar, J. G. B., Oakley. N. W., Spathis, G. S., Sulway, M. J., and Nabarro. J. D. N. (1975). The treatment of acromegaly with special reference to transsphenoidal hypophysectomy. Quarterly Journal of Medicine, New Series, 44, 79-98. 